\title{
Lexis
}

Journal in English Lexicology

1 | 2008

Polysemy

\section{Sens et relations de sens dans les grammaires de construction}

Jérôme Puckica

\section{(2) OpenEdition}

Journals

Édition électronique

URL : http://journals.openedition.org/lexis/767

DOI : 10.4000/lexis.767

ISSN : 1951-6215

Éditeur

Université Jean Moulin - Lyon 3

Référence électronique

Jérôme Puckica, "Sens et relations de sens dans les grammaires de construction », Lexis [En ligne],

1 | 2008, mis en ligne le 04 juillet 2008, consulté le 21 avril 2019. URL : http://journals.openedition.org/ lexis/767 ; DOl : 10.4000/lexis.767

\section{(@) $\Theta \Theta$}

Lexis is licensed under a Creative Commons Attribution-NonCommercial-NoDerivatives 4.0 International License. 


\section{Sens et relations de sens dans les grammaires de construction}

Jérôme Puckica ${ }^{27}$

\section{Résumé}

Cet article étudie la question du sens et des relations de sens dans le cadre des grammaires de construction (GC), en s'intéressant tout particulièrement à la polysémie des signes grammaticaux. Après une brève présentation de ce cadre théorique soulignant notamment l'acception large du terme « sens » dans les GC $(\S 1)$, nous considérerons deux approches des catégories sémantiques $(\$ 2)$. Nous proposerons d'abord une étude critique de l'approche «abstractionniste » du sens suivant laquelle les différents emplois ou sens d'un signe linguistique peuvent être conçus comme des réalisations d'un sens abstrait «invariant» $(\S 2.1)$. Nous nous tournerons ensuite vers la théorie sémantique du prototype adoptée par les GC (§2.2), avant de considérer son application à des constructions grammaticales polysémiques de l'anglais telles que la construction Proposition Ditransitive (§3). Enfin, nous évoquerons brièvement un phénomène que nous proposons de nommer «homomorphie constructionnelle », variante constructionnelle de l'homonymie dans laquelle des structures syntaxiques identiques peuvent être des instances de constructions grammaticales différentes $(\S 4)$.

\footnotetext{
${ }^{27}$ IUFM de Paris.
} 


\section{Les grammaires de construction}

Le courant des grammaires de construction (GC) regroupe un ensemble de modèles grammaticaux qui sont globalement proches des thèses de la linguistique cognitive. Parmi les représentants les plus en vue de ce courant, on peut citer R. Langacker (Cognitive Grammar, 1987, 1991, 1999), Ch. Fillmore et P. Kay (Construction Grammar, 1988, 1993), A. Goldberg (Cognitive Construction Grammar, 1995, 2006) et W. Croft (Radical Construction Grammar, 2001). Sans faire une présentation exhaustive des GC, nous soulignerons ici simplement quelques points importants pour la suite de notre discussion ${ }^{28}$.

Le postulat central des $\mathrm{GC}$ est que le langage verbal humain est fondamentalement symbolique : la connaissance linguistique d'un locuteur natif est essentiellement composée d'un réseau de «constructions », qui sont simplement des signes linguistiques, i.e. des unités symboliques propres à une langue donnée définies par l'association conventionnelle de propriétés formelles et sémantiques. La grammaire est elle-même conçue comme un répertoire d'unités symboliques et donc d'unités porteuses de sens - une conception radicalement opposée à celle, formelle et modulaire, de la grammaire générative.

Les constructions peuvent être classées suivant deux axes gradués. D'une part, suivant un axe horizontal qui oppose un pôle [+simple] à un pôle [+complexe], selon le degré de complexité formelle de la construction : TREE et WORK sont des constructions simples; UNTHINKABLE et ALL OF A SUDDEN, des constructions complexes. D'autre part, suivant un axe vertical qui oppose un pôle [+spécifié] (ou «substantif») à un pôle [+schématique] (ou « formel »), selon que la forme phonologique (voire phonématique) de la construction est plus ou moins spécifiée ou fixée en langue : ALL OF A SUDDEN est une construction complexe et entièrement spécifiée; l'énoncé He pulled her leg présente une instance de la construction PULL NP'S LEG, qui est elle aussi complexe, mais partiellement schématique : en particulier, la position de déterminant du nom LEG est « ouverte », bien que contrainte (seul un déterminant possessif ou un GN au génitif est possible). De même, l'énoncé The less I work, the better I feel est reconnu comme étant une instance de la construction complexe et partiellement schématique conventionnellement notée THE X-ER, THE Y-ER, que Goldberg (2006) nomme Covariational Conditional Construction ${ }^{29}$. Enfin, des énoncés tels que Him be a doctor? et Her write a novel about the Spanish Inquisition?, empruntés à Fillmore et al. (1988), sont des instances d'une construction complexe et entièrement schématique que les auteurs nomment "Construction de Réponse Incrédule » (Incredulity Response Construction). Indépendamment des items lexicaux qu'ils contiennent, les énoncés de ce type présentent un ensemble de propriétés formelles et sémantiques communes et caractéristiques. Du point de vue de la forme : une proposition indépendante de structure déclarative dont le terme sujet est au cas accusatif-objectif (visible avec certains sujets pronominaux), dont le verbe est à la forme infinitive (souvent omis s'il s'agit de BE : e.g. Him, a doctor ?) et qui présente un schéma intonatif interrogatif typique. Du point de vue du sens, ces énoncés ont une valeur illocutoire particulière (question) et contiennent une présupposition : ils expriment une réaction de l'énonciateur qui sous-entend son incrédulité face à une situation (« Je n'arrive pas à le croire !»).

\footnotetext{
${ }^{28}$ D'après Croft \& Cruse (2004:225) : «The cognitive linguistic approach to syntax goes under the name of construction grammar ». En réalité, les divers modèles de GC ne sont pas tous aussi proches de la linguistique cognitive et il existe entre eux des différences considérables. En outre, Langacker tient à distinguer sa « grammaire cognitive» du courant des GC, y compris de la GC « cognitive» de Goldberg (cf. Langacker 2005).

${ }^{29}$ L'emploi de majuscules pour le nom des constructions est conventionnel dans les GC, bien que d'une élégance discutable.
} 
On voit avec ce dernier exemple que l'approche "sémiotique » des GC requiert une acception large du terme " sens », qui reflète en partie ce que la linguistique cognitive nomme la conception « encyclopédique » du sens - principalement, l'idée que l'on ne peut pas isoler les connaissances linguistiques des connaissances extra-linguistiques, et que le sens d'une unité symbolique ne saurait se limiter au « sens du dictionnaire », conçu de façon étroite ${ }^{30}$. Par « sens» (« contenu», voire « fonction» chez certains) d'une construction, on entend non seulement des propriétés sémantiques, mais aussi, le cas échéant, des propriétés qui peuvent être considérées comme pragmatiques, voire discursives ou informationnelles. Par exemple, dans la construction Proposition Ditransitive de l'anglais (ex. He gave her the apple), le référent de l'objet indirect (Récipiendaire) présente ordinairement un degré de thématicité ou topicalité plus élevé que celui de l'objet direct (Thème) : ex. ?*He gave a woman it. Suivant Goldberg (2006), il s'agit là d'une des propriétés du contenu de cette construction.

Les GC estiment qu'il est impossible de fixer objectivement une ligne de séparation nette entre l'idiomatique et le général, l'irrégulier et le régulier ou le lexique et la grammaire : tout est affaire de degrés. En place d'une opposition tranchée lexique v. grammaire, elles reconnaissent un continuum liant deux pôles opposés : le "continuum lexique-grammaire » (ou « lexique-syntaxe »). Le vaste réseau de constructions ainsi reconnu est parfois nommé constructicon (par analogie avec l'anglais lexicon). Les divers schémas de combinaison morphosyntaxique d'une langue sont eux-mêmes des constructions, des "couplages formesens » : par exemple, les divers types de schémas propositionnels fondamentaux de l'anglais [S V O], [S V Oi Od], [S V PCS], etc. - sont conçus comme étant des constructions schématiques complexes qui associent une forme à un sens, indépendamment des items lexicaux qui peuvent y être insérés (cf. $\$ 3.1$ infra) ${ }^{31}$. De ce point de vue, il n'y a pas de différence de nature entre la construction Proposition Ditransitive, la «Construction de Réponse Incrédule », la locution PULL NP'S LEG ou le morphème lexical TREE. Ceci n'exclut évidemment pas des différences qualitatives entre les signes (essentiellement) grammaticaux et les signes (essentiellement) lexicaux : les premiers sont typiquement plus schématiques et abstraits que les seconds du point de vue de leur forme et/ou de leur contenu.

Enfin, les GC ne reconnaissent pas de règles ou principes (morpho)syntaxiques purement formels, autonomes et distincts du sens: les règles grammaticales sont des unités symboliques, tout comme les items lexicaux. En place d'un système où les items lexicaux sont combinés entre eux au moyen de règles ou principes formels, les GC proposent un système où la production concrète d'énoncés repose sur l'assemblage, "intégration » ou " unification» de constructions lexicales et grammaticales, l'unification de deux constructions étant possible par défaut tant qu'il n'y a pas de conflit entre les propriétés de ces constructions (ex. *we drinks, *an information, * sleep the bed). Par ailleurs, toute dérivation ou transformation syntaxique est exclue : les GC sont des modèles dits «non-dérivationnels » ou «surfacistes ». Une proposition passive particulière ne sera pas ici dérivée d'une structure profonde active, mais reconnue comme une instance de la construction Proposition Passive, comme un «construit» dont la production et, inversement, l'interprétation, sont « autorisées » par l'existence de la construction Proposition Passive dans le constructicon.

\footnotetext{
${ }^{30}$ Sur la conception encyclopédique du sens, voir par exemple Langacker (1987) ou Evans \& Green (2006).

${ }^{31}$ Remarquons que [S V O] ou [NP1 V NP2] ne sont que des notations de la forme abstraite de la réalisation canonique de la construction Proposition Transitive Simple (cf. §4 infra).
} 


\section{Deux approches des catégories sémantiques : le modèle des conditions nécessaires et suffisantes $v$. la théorie du prototype}

L'organisation des catégories, tout particulièrement des catégories sémantiques, est un thème central de la sémantique cognitive. Sur ce point, les GC adoptent la théorie du prototype et considèrent que la plupart des signes linguistiques, qu'ils soient lexicaux ou grammaticaux, sont polysémiques. Par opposition, d'autres théories linguistiques adoptent une approche "abstractionniste » du sens inspirée du modèle classique des catégories, le modèle des conditions nécessaires et suffisantes (CNS), et cette approche, dans le cas des signes grammaticaux, équivaut bien souvent à un postulat de monosémie.

\subsection{Le modèle des CNS et l'approche abstractionniste du sens}

Nombre de linguistes prônent une approche du sens que l'on peut qualifier d' " unificatrice» ou d' " abstractionniste» (abstractionist). Cette approche est fondée sur l'hypothèse que les différents emplois ou sens d'un signe linguistique sont liés par un principe sémantique commun plus ou moins abstrait (ou «schématique »); elle consiste ainsi à rechercher l' « invariant sémantique » qui constitue la «valeur fondamentale » d'un signe linguistique - l'unité derrière la diversité ${ }^{32}$.

Si l'on admet que les différents sens d'un signe polysémique, ou polysème, forment une catégorie, on peut alors considérer que l'approche abstractionniste du sens reflète la conception traditionnelle des catégories, i.e. le modèle aristotélicien des conditions nécessaires et suffisantes (CNS) : tous les membres d'une catégorie - ici, les significations d'une unité symbolique - partagent un certain nombre de propriétés, attributs ou traits sémantiques, qui sont des conditions nécessaires et suffisantes pour être membre de la catégorie considérée. Partant, les catégories ont des contours clairs et nets et la catégorisation est une affaire de « tout ou rien », sans degrés possibles (cf. le principe aristotélicien du « tiers exclu $»)^{33}$.

L'approche abstractionniste du sens a mené à des résultats tout à fait intéressants mais n'est pas, toutefois, sans poser de sérieux problèmes au regard des trois points suivants : (1) la logique de l'approche abstractionniste et le traitement de la polysémie ; (2) la schématicité des invariants sémantiques postulés; (3) l'assimilation de l'approche abstractionniste à un postulat de monosémie lorsqu'elle est appliquée à l'étude des signes grammaticaux.

L'argument classique des tenants de l'approche abstractionniste consiste à dire que ce n'est pas le hasard si des sens différents, même s'ils paraissent contradictoires, sont associés à une même forme et "donc» que ces sens ont quelque chose en commun - l'invariant sémantique de la forme considérée. C'est dans ce « donc » que se situe l'erreur, comme l'on

\footnotetext{
${ }^{32}$ Par exemple, dans la théorie psychomécanique de G. Guillaume, tout signe linguistique est associé en langue à un unique «signifié de puissance » qu'il convient de retrouver derrière les multiples « effets de sens » ou «signifiés d'effet» observés en discours, ces derniers pouvant varier selon divers paramètres (cf. Joly \& O’Kelly 1990, Boone \& Joly 1996). De même, Groussier \& Rivière (1996 : 131), dans leur lexique de la Théorie des Opérations Enonciatives (TOE) d'A. Culioli, notent que la « notion » à laquelle renvoie un signe linguistique doit être considérée comme « un dénominateur commun à l'ensemble des significations possibles » de ce signe.

${ }^{33}$ La Théorie des Opérations Enonciatives adopte en fait un modèle de catégorisation qui est comparable à la version standard de la théorie du prototype (cf. infra). Cependant, elle ne semble le faire que pour la description des «domaines notionnels » et non des «notions », si l'on s'en tient à la définition de Groussier \& Rivière (1996) citée dans la note précédente.
} 
peut s'en rendre compte en examinant des cas simples de polysémie lexicale ${ }^{34}$. On peut considérer que les liens qui unissent entre eux les différents sens d'un polysème ne sont jamais le fruit du « hasard » : toute polysémie est motivée et toute extension sémantique dont résulte un nouveau sens d'un signe à partir d'un sens préexistant est motivée, sans pour autant être toujours prévisible. Mais il n'en découle pas qu'il existe nécessairement un dénominateur sémantique commun aux différents sens d'un polysème ${ }^{35}$. Si l'on considère par exemple le nom français NAVET et ses deux sens principaux, (S1) "plante de la famille des crucifères cultivée pour ses racines comestibles » et (S2) « œuvre d'art sans valeur », on note qu'il existe bien un lien entre (S1) et (S2) : (S2) est issu de (S1), "à cause de la fadeur du navet» (Nouveau Petit Robert 2001), mais la notion de fadeur ne saurait être prise comme invariant sémantique du nom NAVET. Le lien entre deux significations - qu'il soit analogique ou métaphorique, métonymique, etc. - ne se confond pas avec un invariant dont celles-ci seraient des instances. De même, s'il existe une explication historique au fait que le nom français BIDON puisse signifier « récipient portatif servant à contenir des liquides » ou «mensonge, tromperie, bluff », on peine à identifier un invariant qui constituerait le « fait explicateur » de ces deux sens ${ }^{36}$. Il apparaît simplement que les divers sens d'un polysème peuvent être liés entre eux sans qu'il existe obligatoirement de propriété qui soit commune à tous (cf. $\$ 2.2$ infra $)^{37}$. On pourrait même arguer qu'il y a polysémie dès lors que le dénominateur commun à deux emplois d'un même signe n'est plus clairement perçu, tout n'étant ici, à nouveau, qu'une affaire de degrés : de la perception d'une nuance de sens, on passe à celle de deux sens distincts mais liés d'un même signe (polysémie), voire, au-delà, à la perception de deux sens distincts sans relation autre que la forme commune qui les exprime (homonymie), indépendamment de leur éventuel lien étymologique.

Le deuxième problème lié à l'approche abstractionniste du sens est celui de la schématicité des invariants sémantiques postulés. A priori, plus grande est la diversité des réalisations sémantiques d'une unité symbolique, plus grande doit être la schématicité ou l'abstraction de l'hypothétique dénominateur sémantique commun à ces réalisations. D'après Kleiber (1999: 58), et malgré nos remarques précédentes à propos des noms NAVET et BIDON, il serait en fait « toujours possible » de trouver un invariant sémantique, "parce que l'on trouve toujours, pour peu évidemment qu'on le désire fortement, des traits abstraits suffisamment élevés pour servir de chapeau aux différents sens que l'on entend rassembler ». Toutefois, il existe alors un risque sérieux pour le grammairien (ou le lexicographe) d'aboutir à un invariant trop schématique et, de ce fait, insuffisamment "prédictif ». Par exemple, en anglais, le morphème grammatical du prétérit simple (-ED) peut être employé pour indiquer qu'un événement est passé, révolu par rapport au moment de parole (ex. John called us last week), mais aussi pour présenter un événement comme fictif, contraire à la réalité du moment présent telle que la conçoit l'énonciateur (ex. I wish I knew the answer). Pour rendre compte à la fois des emplois temporels et modaux du prétérit anglais, on invoque souvent une notion de " distance » (remoteness) ou "décrochage », "rupture », etc., par rapport à la situation d'énonciation $(\mathrm{S} 0)$. On reconnaît alors que le prétérit n'est pas, fondamentalement, une

\footnotetext{
34 L'argument abstractionniste n'est pas censé concerner les cas d'homonymie et notamment les simples coïncidences formelles résultant d'évolutions phonologiques convergentes : ex. angl. nom SOUND1 « son» (du lat. sonus) v. adj. SOUND2 « sain » (du v. angl. gesund). Nous laisserons donc ces cas de côté.

${ }^{35}$ Contrairement à ce qu'avancent par exemple Groussier \& Rivière (1996 : 131) : «si un mot est polysémique, il n'arrive à survivre que s'il demeure un principe commun clair entre ses diverses significations ».

${ }^{36}$ D'après le NPR (2001) et le Trésor de la Langue Française informatisé (http://atilf.atilf.fr/tlf.htm), le sens «mensonge, tromperie, bluff» de BIDON serait issu d'un sens apparu à la fin du XIXe s. et aujourd'hui sorti de l'usage « arg. ensemble de trois coupons de drap pliés de façon à compter pour six », qui était lui-même dérivé du sens premier de BIDON (« récipient portatif $[. .] »$.$) par analogie de forme.$

${ }^{37}$ Groussier \& Rivière (1996: 131) notent même : «si un mot est polysémique, il n’arrive à survivre que s'il demeure un principe commun clair entre ses diverses significations $»$.
} 
marque de temps, mais peut-être une marque de modalité (cf. Lyons 1977, Langacker 1991), et l'on suggère en outre que l'antériorité temporelle et l'irréalité ou contre-factualité ne sont en fait que deux variantes d'un seul et même sens abstrait, donc que le prétérit n'a peut-être en définitive qu'un seul sens ${ }^{38}$. Le problème est que la notion de distance par rapport à S0 paraît trop générale : elle est suffisamment inclusive pour rendre compte des emplois visés, mais elle est également insuffisamment exclusive, car elle n'exclut pas des emplois que n'a pas le prétérit anglais (cf. Taylor 2003 : 180). Par exemple, on s'attendrait a priori à ce que le prétérit puisse s'employer tout aussi bien pour parler d'événements passés que pour parler d'événements futurs, ce qui n'est pas le cas. Par ailleurs, la distance par rapport à S0 exprimée par le prétérit peut être temporelle ou «modale", mais pas spatiale, ni psychologique ou affective, comme celle potentiellement marquée par THAT par opposition à THIS. Suivant les énonciativistes Bouscaren \& Chuquet (1987 : 25), la valeur fondamentale du prétérit serait plus précisément une rupture d'ordre temporel entre le moment de l'énonciation et le moment de l'événement verbal : "entre T0 et le moment du procès, il y a rupture ». Toutefois, demeure le problème du futur ; en outre, la distance modale que peut exprimer le prétérit n'est pas nécessairement aussi une distance temporelle : dans I wish I knew the answer, le moment $\mathrm{du}$ procès qu'exprime knew est présent et non passé (ou futur); c'est une situation contemporaine du moment de parole qui est présentée comme irréelle. Autrement dit, ni la notion de distance par rapport à $\mathrm{S} 0$, ni celle, plus précise, de rupture temporelle entre $\mathrm{T} 0$ et le moment du procès, ne paraît constituer une valeur fondamentale du prétérit à partir de laquelle on pourrait prédire les emplois de ce morphème grammatical : ces hypothèses ne permettent pas une description adéquate du savoir linguistique d'un locuteur anglophone natif. Pour tout invariant postulé dont la «prédictivité » est défaillante, se pose nécessairement la question de sa "réalité psychologique» et donc de son intérêt, la linguistique n'ayant pas pour but de produire des artefacts.

Le troisième et dernier problème que nous voulions souligner à propos de l'approche abstractionniste du sens est qu'elle semble assimilée par beaucoup à une approche monosémique du sens alors qu'il n'y a pas nécessairement d'opposition entre la notion d'invariant sémantique et celle de polysémie. Postuler qu'il existe un dénominateur commun aux différents emplois d'un signe n'équivaut pas à postuler que ce signe est monosémique ${ }^{39}$. Plus généralement, postuler que tout signe linguistique est associé en langue à une valeur fondamentale invariante n'équivaut pas à postuler que tout signe linguistique est monosémique et donc que la polysémie n'existe pas. Pourtant, tout particulièrement dans le cas des signes grammaticaux, il semble que la notion d'invariance soit devenue pour beaucoup synonyme de monosémie. Ainsi, commentant les multiples emplois de l'imparfait français (duratif, itératif, narratif, hypocoristique, irréel, etc.), Wilmet (1998 : 389-90), partisan de la théorie guillaumienne, note : "Une telle disparate suscite deux réactions. Ou l'on se résigne à la polysémie. [...] Ou l'on part à la recherche du fil d'Ariane » - l'invariant sémantique à l'origine des différentes valeurs du signe considéré, ce dernier n'ayant en définitive qu'un seul sens. Tandis que la polysémie des signes lexicaux est largement reconnue comme un état de fait, conclure qu'un signe grammatical est polysémique paraît ici être un pis-aller, un aveu d'impuissance. D'un point de vue théorique, on suggère alors

\footnotetext{
${ }^{38}$ On voit là comment l'approche abstractionniste peut se confondre avec un postulat de monosémie (cf. infra).

${ }^{39}$ La définition de la « notion » par Groussier \& Rivière (1996) citée précédemment (n.5) semble d'ailleurs claire sur ce point : c'est « un dénominateur commun à l'ensemble des significations possibles » d'un signe. Il en va de même avec ce que Langacker (1987) nomme un « schéma », i.e. le membre d'une catégorie qui capture ce qu'il y a de commun à l'ensemble des membres de cette catégorie. Dans le cas d'une catégorie sémantique, ce schéma équivaut à un invariant sémantique, mais il est, en quelque sorte, un sens « supplémentaire » de la catégorie, qui domine tous les autres: sa reconnaissance ne remet aucunement en question l'éventuelle polysémie du signe considéré.
} 
l'existence d'une opposition tranchée entre les signes grammaticaux, qui semblent devoir être monosémiques, et les signes lexicaux, qui sont généralement polysémiques. Cette opposition est manifestement incompatible avec le continuum lexique-grammaire des GC : si le lexique et la grammaire ne sont que deux sous-parties aux frontières floues d'un seul et même réseau d'unités symboliques, si les unités dites lexicales et les unités dites grammaticales sont fondamentalement du même type, i.e. des constructions, alors il n'y a a priori aucune raison pour que la polysémie - ou la monosémie, d'ailleurs - soit une propriété exclusive des signes lexicaux. On pourrait même penser que la polysémie est une propriété attendue des signes grammaticaux: en effet, la polysémie est caractéristique des signes à forte fréquence d'emploi, ce que sont les signes grammaticaux de par leur nature fonctionnelle. De fait, au regard des faits langagiers, la monosémie des signes grammaticaux est une hypothèse dont on cherche les fondements empiriques. Dans le cas du prétérit anglais discuté plus haut, l'antériorité temporelle et l'irréalité paraissent bien être deux sens distincts de ce signe grammatical, ce que tendent à confirmer les différences, importantes, du point de vue de la distribution et de la fréquence en discours, que l'on peut observer entre les emplois temporels et modaux du prétérit (cf. Taylor 2003) : sans plus de contexte, John washed the car ne peut renvoyer qu'à un événement passé ; inversement, John might wash the car ne le peut, pour ainsi dire, pas. Si l'on considère d'ailleurs un auxiliaire modal anglais tel que MAY, l'équipossibilité logique ou contingence (ex. She may be in her room) et la permission (ex. You may take a seat) paraissent bien être, là encore, des sens distincts, pouvant mener à l'ambiguité (ex. He may do it: «Il est possible qu'il le fasse » ou « Il est autorisé à le faire »). Avec MUST (forte probabilité v. obligation), on pourrait également noter des différences distributionnelles au regard de la négation : en anglais britannique, la négation mustn't est habituellement réservée au MUST déontique. On peut également considérer des déterminants tels que SOME, les différents types de génitif, des suffixes dérivationnels tels que -ETTE (ex. KITCHEN-ETTE « small », USHER-ETTE « female », LEATHER-ETTE « imitation ») ; et l'on pourrait encore citer le cas des prépositions, généralement classées parmi les «mots » grammaticaux : les plus courantes sont associées à des dizaines de sens dans les dictionnaires (cf. OVER, §3.2 infra). On peut en fait penser que les divers "mots » et morphèmes grammaticaux d'une langue sont très majoritairement polysémiques : pour ces signes, comme pour les signes lexicaux, la monosémie n'est pas la règle, mais plutôt l'exception. La quête de l'invariant ne saurait donc être assimilée à un postulat de monosémie ; dans la plupart des cas, l'invariant est un sens schématique qui, s'il existe, doit se " superposer » aux autres sens, plus spécifiés, d'un polysème, et non les remplacer.

\subsection{La théorie du prototype et ses deux versions, « standard » et « étendue »}

Les linguistes d'orientation cognitiviste, dont la grande majorité des grammairiens constructionnistes, considèrent que le modèle classique des catégories - le modèle des CNS ne permet pas de rendre compte de la plupart des catégories mentales et donc des catégories linguistiques : au modèle des CNS, qui sous-tend l'approche abstractionniste du sens, ils préfèrent la théorie du prototype, liée aux travaux de la psychologue américaine E. Rosch dans les années 1970 et dont on peut reconnaître, avec G. Kleiber (1990), deux grandes versions : la version «standard » et la version « étendue ».

Suivant la version standard de la théorie du prototype (VSTP), une catégorie est organisée autour d'un prototype, qui en est le membre le plus représentatif, son meilleur exemplaire, éventuellement une idéalisation. La catégorisation d'une entité repose sur l'évaluation de son degré de similarité vis-à-vis de cette référence catégorielle qu'est le prototype. Selon qu'elle est jugée plus ou moins ressemblante au prototype d'une catégorie, qu'elle en partage plus ou 
moins de propriétés, une entité peut ainsi être reconnue comme un membre central (prototypique) ou plus ou moins marginal (périphérique) de cette catégorie. Pour reprendre un exemple classique, si tous les oiseaux sont des oiseaux, il demeure que certains sont conçus comme étant « plus oiseau » que d'autres (ex. moineau v. pingouin), le prototype catégoriel d'un individu pouvant toutefois varier, suivant, par exemple, des facteurs culturels ou géographiques. Une catégorie présente ainsi une organisation radiale, avec un unique centre de gravité auquel sont liés, à des degrés divers, les autres membres de cette catégorie : de fait, il peut n'exister aucune propriété unique, aucune « condition nécessaire » qui soit partagée par l'intégralité des membres d'une catégorie.

Dans la version étendue de la théorie du prototype (VETP), il n'existe plus de prototype catégoriel «global» au sens de la VSTP auquel sont liés tous les membres de la catégorie, mais seulement des « effets de prototypie » et des membres présentant des degrés divers de centralité. Les membres d'une catégorie sont uniquement liés entre eux par des « ressemblances de famille» telles celles évoquées par Wittgenstein (1953) dans sa célèbre discussion des jeux ${ }^{40}$ : chaque membre d'une catégorie possède au moins une propriété en commun avec un autre membre de cette catégorie, mais il peut n'exister, là encore, aucune propriété unique partagée par l'ensemble des membres de la catégorie.

Comme noté par Kleiber (1990 : 162), la version standard de la théorie du prototype en est la «version monosémique » et la version étendue, la «version polysémique ». En outre, la version étendue est bien une extension de la version standard, dans la mesure où elle en intègre plusieurs aspects ${ }^{41}$. Suivant la VETP, une catégorie polysémique ne présente pas de prototype «global», mais chaque sens peut être conçu comme un prototype «local ». Pour reprendre l'exemple du nom NAVET, chacun de ses deux sens - (S1) «plante [...]», (S2) « œuvre d'art sans valeur»- peut être associé à un prototype distinct, la catégorisationdénomination « navet» de telle ou telle plante se faisant ainsi par référence au prototype (S1) et la catégorisation-dénomination "navet» de telle ou telle ouvre d'art se faisant par référence au prototype (S2). En revanche, il ne peut effectivement pas y avoir de prototype commun à $(\mathrm{S} 1)$ et $(\mathrm{S} 2)$ : ce qui est « vraiment » un navet au sens (S1) n'est "vraiment pas » un navet au sens (S2) et inversement. D'autre part, on retrouve dans la VETP la notion de « centralité graduée » dans la mesure où certains sens d'un polysème peuvent présenter un plus haut degré de « saillance psychologique » que d'autres et que l'un d'entre eux peut être perçu comme étant le sens « central » de ce polysème. Ce sens central est également (et peutêtre malheureusement) nommé sens «prototypique » par beaucoup : tel le prototype de la VSTP, il peut en effet être conçu comme étant le membre le plus représentatif de la catégorie s'il est, en particulier, le sens le plus courant du polysème, celui qui est mentionné en premier, etc. ${ }^{42}$ Notons toutefois qu'un polysème peut avoir plusieurs sens dont aucun ne domine

\footnotetext{
${ }^{40}$ Wittgenstein (1953) examine les différents types d'activités nommées « jeux » - jeux de cartes, de table ou plateau (échecs, dames, etc.), jeux de balle, cache-cache, jeux olympiques, etc. - et conclut qu'il n'existe aucune propriété unique qui soit partagée par tous les membres de cet ensemble autre que leur dénomination. Il n'observe que des éléments communs - amusement, règles, compétition, chance, adresse, etc. - différemment distribués : chacune des activités nommée « jeu» ressemble au moins sur un de ces points à une autre activité nommée « jeu », comme le feraient les membres d'une même famille, d'où l'idée que les différents sens du mot « jeu » sont liés entre eux par des « ressemblances de famille ».

${ }^{41}$ Kleiber (1990) voit, quant à lui, dans la VETP une « révision radicale » de la VSTP.

${ }^{42}$ Les critères définitoires de cette centralité sémantique varient selon les auteurs : ainsi, pour justifier le choix de tel ou tel sens d'un polysème comme « central», Goldberg (1995) invoque, parmi d'autres arguments, celui de la simplicité conceptuelle: par exemple, le sens central est un sens concret plutôt qu'abstrait ou métaphorique. Toutefois, le sens le plus courant d'un polysème n'est pas nécessairement son sens le plus simple, comme en témoigne le nom WAY, dont le sens le plus courant est celui, métaphorique, de « manière, moyen » et non son sens littéral premier « chemin, route » (Hudson 1998 : 66). Lakoff \& Brugman (1986), Lakoff (1987), ou Fillmore \& Atkins (2000) semblent considérer, quant à eux, que le sens central d'un polysème est le sens dont
} 
clairement les autres : par exemple, aucun des deux sens du nom français NAVET ne nous paraît clairement plus "saillant» que l'autre ; on pourrait en dire de même de l'adjectif anglais LONG, dont le sens temporel paraît tout aussi saillant que le sens spatial premier dont il est dérivé. Des données statistiques seraient évidemment nécessaires, mais on peut par exemple opposer en français le nom NAVET au nom HUMEUR, dont le sens moderne de « disposition de caractère (passagère) » est manifestement plus courant et saillant que le sens vieilli de « liquide organique du corps humain (ex. bile, mélancolie, sang, etc.) ».

\section{La polysémie constructionnelle}

Pour les grammairiens constructionnistes, les signes grammaticaux, tout comme les signes lexicaux, peuvent être polysémiques et leur polysémie est tout à fait comparable à celle des signes lexicaux. Ainsi, avec certaines constructions propositionnelles de l'anglais telles que la Construction de Mouvement Causé et la construction Proposition Ditransitive, il semble possible de reconnaître un sens central et des sens secondaires ou " périphériques » $(\S 3.1)$, tandis qu'avec d'autres constructions, telles que la préposition OVER et la construction Proposition Intransitive, on peine à identifier un sens véritablement central ou « prototypique » $(\$ 3.2)$.

\subsection{La Construction de Mouvement Causé et la construction Proposition Ditransitive}

Suivant Goldberg (1995), les constructions propositionnelles ou «argumentales » (argument structure constructions) - par exemple, les constructions Proposition Transitive Simple, Proposition Ditransitive, Proposition Transitive Résultative, etc. - sont typiquement polysémiques et non associées à un sens abstrait unique. La linguiste américaine postule que l'on peut généralement reconnaître, pour une construction propositionnelle donnée, un sens central (ou "prototypique ») dont découle un nombre limité de sens liés via des schémas d'extension sémantique plus ou moins récurrents, ou « liens de polysémie » (polysemy links).

On peut illustrer cette approche avec ce que Goldberg (1995) nomme la « Construction de Mouvement Causé » (Caused Motion Construction, ci-après CMC), une construction du type [NP1 V NP2 PP] que l'on pourrait également nommer Proposition Transitive Locative ou de Mouvement. En voici quelques exemples, accompagnés chacun de la glose proposée par l'auteur:

(1) a. She pushed him into the room. [S1 : "X causes Y to move Z"]

b. Sam ordered Bill out of the room. [S2 : Conditions of satisfaction entail "X causes $\mathrm{Y}$ to move Z"]

c. Sam let Bill into the room. [S3 : "X enables Y to move Z"]

d. Harry locked Joe into the room. [S4 : "X causes Y not to move from Z"]

e. He showed them into the room. [S5 : "X helps Y to move Z"]

L'exemple (a) illustre ce que Goldberg (1995) considère être le sens central de la CMC (S1) : un Agent (NP1 sujet, X) cause intentionnellement le mouvement concret d'un Thème

peuvent être dérivés les autres sens de la façon la plus « plausible » et la plus « économique », en synchronie, un argument également repris par Goldberg (1995). Notons simplement qu'il s'agit là d'un troisième critère de centralité qui ne correspond pas nécessairement aux deux autres. 
(NP2 objet, Y) vers un Lieu-But ou suivant un Lieu-Chemin (PP complément, Z), le verbe noyau exprimant typiquement le Moyen de ce mouvement ( $X$ causes $Y$ to move $Z$ by $V$-ing). En (b), avec un verbe de communication, on trouve un premier type d'extension par rapport au sens central, d'ordre métonymique : ici, ce sont les "conditions de satisfaction» de l'événement (acte) exprimé par le verbe qui implique $X$ causes $Y$ to move $Z$; en l'occurrence, l'ordre est "satisfait» (exécuté) si Bill sort de la pièce. En (c), la construction exprime un type particulier de causation, à savoir la permission, voire la levée d'une entrave. En (d), on passe de l'idée de causer la réalisation d'un événement à celle de causer sa non-réalisation : la construction exprime, tout au contraire, une entrave ou un blocage. Enfin, en (e), la CMC exprime un autre type de causation, qui est l'aide ou facilitation.

Chacun des sens de la CMC est "instancié » par une sous-classe de verbes. Pour en donner quelques exemples, (S1) est instancié par PUSH, KICK, SHOVE, etc. ; (S2) par ORDER, ASK, INVITE, etc. ; (S3) par ALLOW, LET, FREE, etc. ; (S4) par LOCK, KEEP, BARRICADE, etc. ; et (S5) par HELP, SHOW, GUIDE, etc. Si les diverses gloses proposées sont certainement critiquables, on conçoit néanmoins assez aisément les liens sémantiques entre les différents sens de la CMC. Que certains de ces sens soient contraires n'est pas, en soi, un phénomène inhabituel : il n'est pas si rare qu'un mot puisse signifier une chose et son contraire, ou du moins une relation inverse ${ }^{43}$. Goldberg (2006: 28), s'appuyant notamment sur des études psycholinguistiques, note de même : " it is clear that both the negation and the antonym of a particular concept are closely associated with that concept ».

Goldberg (1995) justifie notamment la polysémie (limitée) des constructions grammaticales en arguant que celle-ci évite une polysémie rampante des unités lexicales, et donc - non sans une certaine ironie - au nom d'un principe d'économie cher à la grammaire générative, qui a pourtant éliminé de sa théorie la notion de construction grammaticale (Chomsky 1995), mais aussi au nom d'un principe de " plausibilité » des sens verbaux, qui peut paraître sujet à caution. Pour illustrer cette "polysémie rampante » à laquelle nous faisons allusion, on peut considérer les énoncés suivants :

(2) a. If she were acting now, people would probably laugh her off the stage. $\left(\mathrm{CCCS}^{44}\right)$

b. Pat sneezed the napkin off the table (Goldberg 1995).

Suivant l'approche grammaticale traditionnelle, le sens d'une proposition verbale est déterminé par son verbe-noyau. Pour rendre compte des exemples ci-dessus, il faudrait donc reconnaître que les verbes LAUGH et SNEEZE peuvent, dans un de leur sens, être glosés « $X$ causes $Y$ to move $Z$ by [laughing / sneezing] ». Pour de très nombreux verbes pouvant être employés dans la CMC, il faudrait ainsi prévoir un sens supplémentaire, qui parait parfois assez peu "plausible » selon Goldberg, tel le sens «causer le déplacement d'un objet en éternuant (dessus) » pour SNEEZE. Par ailleurs, il faudrait éventuellement ajouter autant de sens supplémentaires que de constructions dans lesquelles le verbe peut être employé : par exemple, un autre sens de LAUGH devrait être postulé pour rendre compte d'énoncés tels que He laughed his way out of the court et encore un autre pour She laughed herself stupid at the idea (cf. Puckica 2007b). Suivant l'approche constructionniste, le sens d'une proposition verbale n'est que partiellement déterminé par son noyau verbal : la construction avec laquelle ce verbe est combiné a son propre contenu sémantique et apporte ainsi sa propre contribution

\footnotetext{
${ }^{43}$ Par exemple : fr. HÔTE ( « qui donne l'hospitalité » ou « qui reçoit l'hospitalité »), fr. LOUER (« donner à loyer » ou « prendre à loyer »), angl. SANCTION (« approuver, permettre » ou « condamner, interdire »), angl. CLIMB (« monter » (climb up) ou « descendre » (climb down)).

${ }^{44}$ Cobuild Concordance and Collocations Sampler, http://www.collins.co.uk/Corpus/CorpusSearch.aspx
} 
à l'interprétation globale de la proposition. Partant, il n'est pas nécessaire de postuler un sens particulier des verbes LAUGH et SNEEZE pour rendre compte des énoncés ci-dessus : le sens « $X$ causes $Y$ to move $Z$ by [laughing / sneezing] » est un produit de la combinaison du sens du verbe impliqué et du sens de la $\mathrm{CMC}$, les énoncés eux-mêmes résultant de la combinaison (unification, intégration) de ces verbes avec la CMC.

Suivant Goldberg (1995), les « liens de polysémie » sur lesquels repose la polysémie des constructions propositionnelles sont des schémas d'extension sémantique (métaphoriques, métonymiques, etc.) plus ou moins récurrents ${ }^{45}$. La polysémie de ces signes grammaticaux est en cela comparable à la polysémie des signes lexicaux, qui repose également en partie sur des métaphores et métonymies plus ou moins régulières ${ }^{46}$. Par exemple, les liens qui unissent le sens central de la CMC à ses autres sens se retrouvent avec la construction Proposition Ditransitive de l'anglais, chaque sens de cette dernière étant à nouveau instancié par une sousclasse de verbes :

(3) a. Joe gave Sally the ball. [S1 :"X causes Y to receive Z" (central sense)]

b. Joe promised Bob a car. [S2 : Conditions of satisfaction entail "X causes Y to receive Z"]

c. Joe permitted Chris an apple. [S3 : "X enables $\mathrm{Y}$ to receive Z"]

d. Joe refused Bob a cookie. [S4 : "X causes Y not to receive $Z "]$

e. Joe baked Bob a cake. [S5 : "X intends to cause $Y$ to receive $Z "]$

f. John bequeathed Bob a fortune. [S6 : "X acts to cause $\mathrm{Y}$ to receive $\mathrm{Z}$ at some future point in time"]

On peut remarquer que l'extension livrant le sens (S5) de la CMC - le passage de l'idée de causer directement (le mouvement de Y) à celle d'aider ou faciliter (ce mouvement) - ne se retrouve pas avec la construction Proposition Ditransitive : face à She helped him out of the room: "Elle l'a aidé à sortir de la pièce », on ne trouve pas * She helped him the prize pour signifier «Elle l'a aidé à obtenir le prix » (cf. Goldberg 1995: 76). Inversement, les extensions livrant les sens (S5) et (S6) de la construction Proposition Ditransitive ne se retrouvent pas avec la CMC, qui ne possède pas de sens correspondants. Les liens de polysémie ne sont donc que partiellement réguliers et les différents sens d'une construction propositionnelle doivent de fait être appris, comme on apprend les différents sens d'un signe lexical.

Enfin, toujours suivant Goldberg (1995), les emplois de la CMC ou de la construction Proposition Ditransitive qui ne correspondent pas aux sens détaillés ci-dessus sont typiquement «sanctionnés » par l'existence de métaphores conceptuelles (Lakoff \& Johnson 1980) dont l'existence peut être démontrée indépendamment de ces constructions. Par exemple, dans le cas de la construction Proposition Ditransitive, le passage du transfert concret d'un objet physique dans He gave her an apple à un transfert abstrait dans He gave her that idea ou He told her a secret reposerait sur ce que Reddy (1979) nomme la « métaphore du conduit» (conduit metaphor), une métaphore que Lakoff \& Johnson (2003 [1980] : 10) résument de la façon suivante :

\footnotetext{
${ }^{45}$ Cf. Goldberg (1995: 164) : «It seems that patterns of constructional extension, like patterns of polysemy generally, embody subregularities (Wilenski 1991); that is, patterns of polysemy recur, although not strictly predictably so $\gg$.

${ }^{46}$ Cf. Apresjan (1974). Un exemple de polysémie « régulière » des unités lexicales est la relation métonymique par laquelle le nom d'un créateur (auteur, peintre, sculpteur, etc.) peut être employé par extension pour désigner une réalisation (œuvre) de ce créateur (ex. acheter un Picasso, le dernier John Grisham). Autre exemple : la métonymie contenant-contenu (ex. boire un verre, finir la bouteille, avaler une cuillère de sirop).
} 
IDEAS (or MEANINGS) ARE OBJECTS. LINGUISTIC EXPRESSIONS ARE CONTAINERS. COMMUNICATION IS SENDING. The speaker puts ideas (objects) into words (containers) and sends them (along a conduit) to a hearer who takes the idea/objects out of the word/containers.

Cette métaphore se retrouve par exemple dans :

(4) a. He put that idea [into words / on paper / into her head / to her]. b. He got the idea across to her. c. His song [captures / conveys] this idea very well. d. "Bush's hollow words on climate change" (The Independent, 29/09/2007) e. I didn't take in much of what she said. f. I don't get it.

En bref, au regard de constructions telles que la CMC ou la construction Proposition Ditransitive de l'anglais, il semble non seulement que les signes grammaticaux puissent, comme les signes lexicaux, être polysémiques, mais aussi que leur polysémie, impliquant des schémas d'extension sémantique partiellement réguliers, soit comparable à celle des signes lexicaux. En outre, certaines métaphores conceptuelles semblent impliquées à la fois dans des emplois métaphoriques de signes lexicaux et de signes grammaticaux. On peut voir là des arguments qui tendent à confirmer l'hypothèse constructionniste d'un continuum lexiquegrammaire.

\subsection{La préposition OVER et la construction Proposition Intransitive}

Tout comme certains polysèmes lexicaux (ex. l'adjectif anglais LONG ou le nom français NAVET), certains signes grammaticaux polysémiques ne possèdent pas de sens qui soit clairement central ou " prototypique ». C'est par exemple le cas de la préposition OVER, qui a fait l'objet de nombreuses études (ex. Brugman 1981, Lakoff 1987, Taylor 2003 ${ }^{47}$ ):

(5) a. The panting is over the mantle.

b. The plane flew over the hill.

c. A white sheet lay over the body.

d. He walked over the hill.

e. He jumped over the wall.

f. He lives over the hill.

g. Will you be home over the summer vacation?

Les études mentionnées s'accordent à reconnaître qu'il n'existe pas de dénominateur commun aux différents sens de OVER, dont (5) ne livre qu'un petit échantillon ${ }^{48}$; elles ne reconnaissent que des ressemblances de famille qui lient chaque sens de cette préposition à un autre de ses sens - tout comme l'avait fait Wittgenstein (1953) à propos des différents sens du mot « jeu ». En (a), OVER exprime une relation statique entre une entité repère (Figure, ciaprès EF) et une entité repérée (Ground, ci-après EG) : EF est située au-dessus de EG et n'est pas en contact avec EG. En (b), on retrouve ce sens «au-dessus et pas en contact», mais OVER exprime cette fois un mouvement (dynamique) de EF qui parcourt EG, et non une

\footnotetext{
${ }^{47}$ Plusieurs exemples et autres éléments de cette présentation des sens de OVER sont empruntés à Lakoff (1987) et Taylor (2003).

${ }^{48}$ En particulier, cet échantillon n'inclut pas d'emplois dits «adverbiaux » de OVER (ex. He turned the page over / The discussion is over). Pour de nombreux linguistes (cognitivistes et autres), OVER demeure en fait une préposition (intransitive) dans ces emplois et ces derniers doivent donc être inclus dans la description sémantique de la préposition OVER.
} 
position (statique). En (c), OVER signifie «placé au-dessus et en contact». On retrouve en partie ce sens en (d), si ce n'est que, comme pour la relation (a)-(b), l'on passe d'une position à un mouvement et qu'en (d) s'ajoute en plus l'idée que la trajectoire de EF est courbe. En (e), « Il sauta par-dessus le mur », on retrouve des éléments de sens de (d) avec en plus l'idée que EG constitue un obstacle à franchir sur la trajectoire (courbe) de EF, etc. En (f), « Il vit de l'autre côté de la colline », OVER ne désigne plus la trajectoire de EF mais le point final de la trajectoire à suivre pour atteindre $\mathrm{EG}$, etc.

Si les études mentionnées s'accordent sur certains points, elles diffèrent cependant quant à ce qui pourrait être le sens central de OVER. Des dictionnaires tels que le Longman Dictionary Of Contemporary English (2003), dont les entrées ne sont pas organisées chronologiquement, citent généralement en premier les sens illustrés par les énoncés (a) et (b), sans faire de distinction entre les notions de position et de mouvement. D'après Lakoff (1987 : 417), Brugman (1981) retient plus précisément le sens (b) comme sens central (« the above-across sense »). Mais même si l'on omet cette distinction entre position et mouvement et que l'on ne retient que le sens « vertical to, not in contact with », se pose alors, suivant Taylor (2003 : 118-19), le problème que ce sens "prototypique » de OVER serait presque identique au sens central de ABOVE; or, suivant la théorie du prototype, les prototypes respectifs de ces deux catégories devraient être clairement distincts ${ }^{49}$. Suivant Taylor (id.), c'est en fait le sens illustré par (e) qui est le sens le plus courant de OVER, et c'est donc ce sens qui devrait être considéré comme central, ce qui règlerait ainsi le problème théorique soulevé.

La construction Proposition Intransitive de l'anglais pose un problème comparable. Nombre de linguistes s'accordent sur l'existence d'un " événement transitif prototypique » qui, du point de vue constructionniste, constituerait le sens central ("prototypique ») de la construction Proposition Transitive Simple: l'action ponctuelle et concrète d'un Agent volontaire sur un Patient qui est pleinement affecté par cette action (cf. Hopper \& Thompson 1980). En revanche, on peine à identifier ce que pourrait être l'hypothétique événement intransitif prototypique. Comme noté par Tsunoda (1994: 4677) :

It is not clear what type of IntrCls [intransitive clauses] are best considered ProtoIntrCls [prototypical intransitive clauses]. [...] Regarding the semantic aspect, for instance, IntrCls expressed varied meanings, such as activity, e.g. [He sat down], change, e.g. [Kim died yesterday], state, e.g. [Many problems remain], etc. Do these meanings represent different degrees of semantic intransitivity? Can any of them be singled out as the semantic property of ProtoIntrCls ?

Un problème, nous semble-t-il, dans cette quête, peut-être illusoire, d'un prototype intransitif «global», est que l'on regroupe sous une seule et même étiquette - «Proposition Intransitive » (ci-après P.I.) - plusieurs constructions intransitives, dont la construction P.I. Simple type [NP V] (ex. John works), la construction P.I. Attributive type [NP V AP] (ex. Sarah is tall) et la construction P.I. Locative (ou de Mouvement) type [NP V PP] (ex. Sarah rushed into the room). Or, il s'agit là de trois constructions distinctes. D'ailleurs, Langacker (1999 : 25-6) note que ce que nous nommons les constructions P.I. Attributive et P.I. Locative ont pour origine deux « archétypes conceptuels » différents, respectivement : « the archetypal conception of an object simply being in a certain state » et « the archetype of a physical object moving through space ». Parallèlement, on remarquera que les différents types de constructions transitives ne sont, eux, pas confondus : par exemple, on ne confond pas la construction Proposition Transitive Simple type [NP1 V NP2] avec la Proposition Transitive

\footnotetext{
${ }^{49}$ Plus précisément, c'est parce que OVER et ABOVE sont reconnus comme étant des termes ou catégories « du niveau de base » (basic level categories) que leurs prototypes devraient être clairement distincts (cf. Taylor id.).
} 
Locative (CMC) type [NP1 V NP2 PP], ni avec la construction Proposition Ditransitive type [NP1 V NP2 NP3]. Chacune de ces constructions est définie par un ensemble de propriétés formelles et sémantiques unique qui justifie sa reconnaissance comme unité symbolique de la langue ; chacune semble avoir son propre prototype sémantique (sens central), mais rien ne garantit l'existence d'une construction «Proposition Transitive » plus générale dont elles seraient des instances ${ }^{50}$. Que ce « super-schéma » transitif existe ou non dans le constructicon reste, nous semble-t-il, à démontrer. Peut-être ce super-schéma n'aurait-il d'ailleurs qu'une importance cognitive et linguistique négligeable (cf. Langacker 1987 : 381). En tout état de cause, on ne voit pas quel pourrait être le prototype de cette construction hypothétique. De fait, nous ne nous étonnons pas que l'on ne parvienne pas non plus à déterminer clairement un prototype « intransitif ».

Si tant est que l'on puisse reconnaître une construction Proposition Intransitive dont les constructions P.I. Simple, P.I. Attributive et P.I. de Mouvement seraient des sous-types, il reste toutefois une solution théorique : postuler que cette construction est polysémique et qu'elle ne présente pas de sens clairement central mais seulement des sens plus ou moins saillants liés entre eux par des ressemblances de famille. La notion de mouvement est en effet liée à celle de changement, qui à son tour permet de passer de la notion d'état à celle de changement d'état, etc. ${ }^{51}$ Solution de facilité ? Telle l'analogie des néogrammairiens, la version étendue de la théorie du prototype prête le flanc à la critique : elle peut, semble-t-il, tout expliquer, ce qui, pour certains, signifiera qu'elle n'explique rien du tout. Cependant, la théorie du prototype dans son entier est fondée sur l'idée qu'il existe très peu de contraintes pesant sur les possibilités d'association au sein d'une catégorie sémantique donnée. Comme le laisse entendre Taylor (2003: 122), " practically anything can get associated with anything else within a category ». Des polysèmes tels que les noms français NAVET («plante [...]» ou « oeuvre d'art sans valeur ») ou BIDON (« récipient portatif [...]» ou « mensonge, tromperie, bluff ») illustrent le caractère à la fois motivé et imprévisible de certaines extensions sémantiques, de même que l'exemple souvent cité des classificateurs nominaux en dyirbal, dont l'hétérogénéité sémantique est à l'origine du titre Women, Fire, and Dangerous Things de Lakoff $(1987)^{52}$. Une fois encore, s'il existe bien un continuum lexique-grammaire, on peut s'attendre à ce que le contenu sémantique de certains signes grammaticaux présente des propriétés comparables à celui de signes lexicaux.

\section{L'homomorphie constructionnelle}

Une dernière note s'impose avant de conclure cette étude : il apparaît souvent qu'à une structure syntaxique donnée peuvent correspondre des types d'événements sensiblement différents. Par exemple, les deux énoncés ci-dessous présentent une même structure syntaxique du type [NP1 V NP2 PP] :

\footnotetext{
${ }^{50}$ Rien ne garantit non plus l'existence d'un invariant schématique pertinent pour chacune de ces constructions. Par ailleurs, notons bien que par « Proposition Transitive» nous n'entendons pas ici la construction Proposition Monotransitive ou Transitive Simple (type [NP1 V NP2] ou [SVO]), mais bien une (hypothétique) construction plus générale qui, dans le constructicon, dominerait cette dernière et les autres mentionnées.

${ }^{51}$ Kemmer (2006), exploitant l'étude typologique de Stassen (1997) sur les constructions intransitives, souligne ces relations entre état, action, changement et mouvement, mais conclut quant à elle qu'il existe bel et bien un prototype intransitif, qui serait une notion de mouvement.

${ }^{52}$ Le dyirbal est une langue aborigène d'Australie dont les classificateurs nominaux ont été notamment étudiés par Dixon (1982) et Lakoff (1987).
} 
(6) a. She put the newspaper in the basket. b. I consider him as a friend.

La structure [NP1 V NP2 PP] peut exprimer l'action d'un Agent qui cause le mouvement d'un Thème vers un Lieu-But (a) mais on la trouve également avec des verbes exprimant une opinion ou appréciation, comme en (b). Le complément prépositionnel serait d'ailleurs différemment analysé : en (a), il exprime le lieu où se trouve le référent de l'objet au terme de l'événement; en (b), il exprime un attribut de l'objet (I consider that he is a friend). De fait, une notation fonctionnelle plutôt que catégorielle de la structure syntaxique des énoncés (a-b) refléterait cette différence : [S V O LCO] pour (a) et [S V O PCO] pour (b) ${ }^{53}$. Autrement dit, tandis que (a) est une instance de la CMC ou construction Proposition Transitive Locative, (b) est une instance de ce que l'on peut nommer la construction Proposition Transitive Attributive.

Dans l'hypothèse d'un continuum lexique-grammaire, ce phénomène n'a rien d'étonnant. Des formes (syntaxiques) identiques peuvent être des instances de constructions différentes, comme c'est le cas pour des unités lexicales simples (homonymes). En réalité, ce phénomène doit même être attendu : si le lexique contient des dizaines de milliers d'unités symboliques formellement différentes, les constructions propositionnelles d'une langue sont, elles, assez peu nombreuses. C'est donc sans surprise que l'on trouve ce que l'on pourrait nommer des constructions « homomorphes » (pour éviter le terme « homonyme », ici peu adapté).

Cette homomorphie n'est d'ailleurs aucunement limitée à des constructions propositionnelles schématiques telles que la CMC. En anglais, He took the biscuit peut tout aussi bien être une instance de la locution ou expression idiomatique TAKE THE BISCUIT ( «Il a décroché le pompon ou la timbale ») qu'une combinaison syntaxique libre (« Il a pris le biscuit $»)$. De même, en français, Fred a pris le taureau par les cornes peut tout aussi bien être une instance de la locution PRENDRE LE TAUREAU PAR LES CORNES qu'une combinaison syntaxique libre, livrant deux interprétations sémantiques bien différentes («Fred a pris de front les difficultés » v. «Fred a saisi le bovidé mâle par les excroissances osseuses situées sur sa tête »).

L'existence d'une homomorphie constructionnelle implique que l'épineux problème de la distinction entre polysémie et homonymie se pose également au niveau des constructions complexes, et non seulement au niveau des unités lexicales simples.

Enfin, on notera que des structures telles que [NP1 V NP2 PP] ou [S V O LCO] ne sont que des notations de la forme abstraite de la réalisation canonique de la CMC. Par exemple, l'ordre des constituants d'une instance de cette construction peut varier si celle-ci est combinée avec une construction interrogative ou relative :

(8) a. What did she put in the basket? b. This is the newspaper [that she put in the basket].

Cette «allomorphie» constructionnelle implique à son tour que la forme d'une construction telle que la $\mathrm{CMC}$ dans le constructicon est nécessairement plus schématique et abstraite que la structure qui en est la réalisation canonique et prototypique (cf. Michaelis 2006, Puckica 2007a).

\footnotetext{
${ }^{53}$ L'étiquette « $\mathrm{LCO}$ » désigne un complément locatif, l'indice « $\mathrm{O}$ » notant le rapport avec l'objet (puisque ce complément exprime typiquement le lieu final du référent de l'objet et non du sujet). L'étiquette « PCO » est, quant à elle, couramment utilisée pour la fonction d'attribut de l'objet (Object Predicative Complement).
} 


\section{Conclusion}

Suivant l'approche du langage proposée par les grammaires de construction, la connaissance linguistique d'un locuteur natif est essentiellement constituée d'un réseau de constructions, i.e. d'unités symboliques définies par l'association de propriétés formelles et sémantiques. Le "sens " d'une construction peut inclure ici tout à la fois des propriétés sémantiques et pragmatiques voire discursives ou informationnelles. Par ailleurs, les diverses règles et catégories grammaticales sont elles-mêmes des unités symboliques et non des entités purement formelles. De ce fait, il n'existe pas d'opposition tranchée entre le lexique et la grammaire, mais seulement un "continuum lexique-grammaire ». Bien que l'on reconnaisse des différences qualitatives entre les unités symboliques " lexicales» et " grammaticales », celles-ci sont fondamentalement du même type et il n'y a donc pas de raison a priori pour que la polysémie (ou la monosémie) soit une propriété exclusive des unes ou des autres.

Certaines théories linguistiques adoptent une approche abstractionniste du sens suivant laquelle un signe linguistique est associé à un invariant sémantique abstrait qu'il convient de retrouver derrière la diversité de ses réalisations en discours. Inspirée du modèle de catégorisation classique des conditions nécessaires et suffisantes, cette approche pose divers problèmes : elle suit une logique discutable qui semble remise en question par des cas simples de polysémie lexicale; les invariants sémantiques qu'elle postule paraissent parfois trop généraux et insuffisamment prédictifs, ce qui pose la question de leur réalité psychologique et donc de leur intérêt; enfin, la quête de l'invariant équivaut bien souvent à un postulat de monosémie dans le cas des signes grammaticaux, postulat à la fois contraire à l'approche constructionniste et, semble-t-il, à la réalité des faits langagiers. Par opposition, les grammaires de construction admettent volontiers la polysémie des signes grammaticaux et préfèrent, quant à elles, la théorie sémantique du prototype. Appliquée à des catégories sémantiques polysémiques, cette théorie suggère qu'il peut n'y avoir aucune propriété commune aux différents sens d'un polysème, qui sont liés entre eux par des « ressemblances de famille ». Les sens-membres d'une catégorie polysémique peuvent présenter des degrés divers de centralité : on trouve ainsi des signes ou constructions qui présentent un sens central ou « prototypique » et des sens secondaires et d'autres pour lesquels il n'existe pas clairement de sens central, mais seulement plusieurs sens plus ou moins saillants - et ce, indépendamment de la nature essentiellement lexicale ou essentiellement grammaticale de l'unité considérée. Si l'on considère en outre la relative régularité de certains cas de polysémie grammaticale et les emplois de métaphores conceptuelles communs à des signes lexicaux et grammaticaux, il apparait alors que la polysémie des signes grammaticaux est en de nombreux points comparable à la polysémie des signes lexicaux, constat qui tend donc à confirmer que le lexique et la grammaire, loin de former deux composants distincts, s'inscrivent tout au contraire dans un continuum d'unités symboliques.

\section{Références}

Apresjan, J. 1974. "Regular polysemy". Linguistics, 142, pp. 5-33.

Boone, A. \& Joly, A. 1996. Dictionnaire terminologique de la systématique du langage. Paris : L'Harmattan.

Bouscaren, J. \& Chuquet, J. 1987. Grammaire et textes anglais. Guide pour l'analyse linguistique. Gap / Paris : Ophrys. 
Brugman, C. 1981. Story of Over. M.A. Thesis. University of California, Berkeley.

Croft, W. 2001. Radical Construction Grammar. Oxford : OUP.

Croft, W. \& Cruse, A.D. 2004. Cognitive Linguistics. Cambridge : CUP.

Dixon, R.M.W. 1982. Where Have All the Adjectives Gone? And Other Essays in Semantics and Syntax. Berlin : Mouton de Gruyter.

Evans, V. \& Green, M. 2006. Cognitive Linguistic: An Introduction. Mahwah, N.J. \& London : Lawrence Erlbaum Associates.

Fillmore, Ch. J., Kay, P., \& O’Connor, M.C. 1988. "Regularity and Idiomaticity in Grammatical Constructions: The Case of Let Alone". Language, 64, 3, pp.501-538.

Fillmore, Ch. J. \& Kay, P. 1993, 1995. Construction Grammar Coursebook. Manuscript, University of California at Berkeley, Department of Linguistics.

Fillmore, Ch. J. \& Atkins, B.T.S. 2000. "Describing polysemy: the case of 'crawl"' In Ravin, Y. \& Leacock, C. (eds.), Polysemy: Theoretical and Computational Approaches, Oxford : OUP, pp.91-110.

Fodor, J.A. 1983. The Modularity of Mind. Cambrdige, Ma. : MIT Press.

Fried, M. \& Östman, J.-O. 2004. "Construction Grammar: A Thumbnail Sketch". In Fried, M. \& Östman, J.-O. (eds.) Construction Grammar in a Cross-Language Perspective, Amsterdam / Philadelphia : J. Benjamins, pp.11-86.

Goldberg, A.E. 1995. Constructions: A Construction Grammar Approach to Argument Structure. Chicago and London: The University of Chicago Press.

---, 2003. "Constructions: a new theoretical approach to language". In : Trends in Cognitive Sciences, 7, 5, pp. 219-224.

---, 2006. Constructions at Work. Oxford : OUP.

Groussier, M.-L. \& Rivière, C. 1996. Les mots de la linguistique. Lexique de linguistique énonciative. Gap / Paris : Ophrys.

Hopper, P.J. \& Thompson, S.A. 1980. "Transitivity in Grammar and Discourse". Language, 56, 2, pp. 251-299.

Hudson, J. 1998. Perspectives on fixedness: applied and theoretical. Lund Studies in English, 94. Lund : Lund University Press.

Joly, A. \& O’Kelly, D. 1990. Grammaire systématique de l'anglais. Structures fondamentales. Paris : Nathan Université.

Kay, P. \& Fillmore, Ch.J. 1999. "Grammatical constructions and linguistic generalizations: the What's X doing Y? Construction ». Language, 75, 1, pp.1-33.

Kemmer, S. 2006. "Intransitive Structures and Functions". Communication. $3^{\text {rd }}$ International BAAHE Conference. Leuven (Belgique), 7-9 décembre 2006.

KLeIBer, G. 1990. La sémantique du prototype. Catégories et sens lexical. Paris : PUF.

---, 1999. Problèmes de sémantique : la polysémie en questions. Villeneuve d'Ascq : Presses Universitaires du Septentrion.

Lakoff, G. 1987. Women, Fire, and Dangerous Things: What Categories Reveal about the Mind. Chicago / London : The University of Chicago Press.

Lakoff, G. \& Brugman, C. 1986. “Argument forms in lexical semantics”. BLS, 12, pp.442-54.

Lakoff, G. \& Johnson, M. 2003 [1980]. Metaphors We Live By. Chicago / London: The University of Chicago Press. 
Langacker, R.W. 1987. Foundations of Cognitive Grammar. Vol. 1: Theoretical Prerequisites. Stanford, Ca. : Stanford U.P.

---, 1991. Foundations of Cognitive Grammar. Vol. 2 : Descriptive Application. Stanford, Ca. : Stanford U.P.

---, 1999. Concept, Image, and Symbol. The Cognitive Basis of Grammar. Berlin, New York : Mouton de Gruyter.

---, 2005. "Integration, grammaticization, and constructional meaning”. In : Fried, M. \& Boas, H.C. (eds.) Grammatical Constructions. Back to the Roots, Amsterdam / Philadelphia : J. Benjamins, pp.157-190.

Lyons, J. 1977. Semantics, volume 2. Cambridge : CUP.

Mathieu, Yvette Y. 2003. " La grammaire de construction ». In : Linx, 48, pp. 43-56.

Michaelis, L.A. 2006. 2006. "Construction Grammar”. In : Brown, K. (ed.) Encyclopedia of Language and Linguistics, 2nd edition, Boston, Ma. : Elsevier, pp.73-84.

Puckica, J. 2003. La diathèse en anglais contemporain : formes, valeurs et sens. Essai de redéfinition d'une catégorie grammaticale. Thèse de doctorat. Bordeaux: Univ. Bordeaux 3.

---, 2006. «La valence : de Tesnière à la sémantique des cadres ». Communication dans le cadre du séminaire "Lexique-Syntaxe" du laboratoire LIDILEM, Univ. Grenoble 3, 24/03/2006.

---, 2007a. "Au-delà de l'allomorphie : variation et représentation formelles dans les grammaires de construction ». Communication dans le cadre du séminaire "LexiqueSyntaxe » du laboratoire LIDILEM, Univ. Grenoble 3, 01/06/2007.

---, 2007b. « Les grammaires de construction ». Anglophonia, 22.

Ravin, Y. \& Leacock, C. (eds.) 2000. Polysemy: Theoretical and Computational Approaches. Oxford : OUP.

Stassen, L. 1997. Intransitive predication: An essay in linguistic typology. Oxford : OUP.

TAYLOR, John R. 2003[1989]. Linguistic Categorization. 3rd edition. Oxford : OUP.

Tournier, J. 1993. Précis de lexicologie anglaise. 3e édition, mise à jour et corrigée. Paris : Nathan Université.

Tsunoda, T. 1994. "Transitivity”. In : Asher, R. \& Simpson, J. (eds.) The Encyclopedia of Language and Linguistics, vol. 9., Oxford : Pergamon Press, pp. 4670-4677.

WiLmet, M. 1998. Grammaire critique du français. 2e édition. Bruxelles : Hachette / Duculot. Wittgenstein, L. 1953. Philosophical Investigations. New York : Macmillan. 